\title{
Magnetoelastic Properties of Hitperm-Type Alloys from Torque Moment
}

\author{
J. Salach, A. Bieńkowski and R. Szewczyk \\ Institute of Metrology and Measurement Systems \\ Warsaw University of Technology \\ św. A. Boboli 8, 02-525 Warszawa, Poland
}

\begin{abstract}
The following paper presents the results of the investigation on the influence of the torque on the magnetic characteristics of the Hitperm-type $\mathrm{Fe}_{41.5} \mathrm{Co}_{41.5} \mathrm{Cu}_{1} \mathrm{Nb}_{3} \mathrm{~B}_{13}$ alloy, in as-quenched and nanocrystalline state. The methodology of application of the torque to the ring-shaped core is also described. This methodology gives a possibility of achieving uniform shearing stresses in the core. The results indicate that shearing stress sensitivity of Hitperm-type alloys depends on the annealing and nanocrystallization process. This effect should be considered during the development of magnetoelastic torque sensors.
\end{abstract}

PACS numbers: 81.07.Bc, 75.80.+q

\section{Introduction}

Previous papers showed that amorphous and nanocrystalline soft magnetic alloys exhibit high magnetoelastic sensitivity, together with high mechanical strength [1]. As a result, these materials can be utilized in development of the magnetoelastic sensors for compressive stress detection [2]. Very promising results were achieved in application of such sensors for monitoring of locomotives. On the other hand, the possibility of application of amorphous and nanocrystalline alloys as torque sensors still seems not to be properly exploited. This fact is mainly caused by the lack of sufficient knowledge about torque dependence of magnetic characteristics of amorphous and nanocrystalline alloys.

\section{Method of investigation}

Previously presented methods of testing of the influence of torque on the magnetic characteristics of amorphous and nanocrystalline alloys are not suitable for magnetoelastic sensors development. In these investigations, amorphous alloy 
strips were glued to a non-magnetic shaft [3]. As a result, the small value of the cross-section of the metallic glass strip caused low values of signal from the sensor, leading to unbeneficial signal to noise ratio. Moreover, due to the fact that magnetic circuits of the strips are open, demagnetization energy appears in the total balance of the free energy of sensing core. This phenomenon leads to a significant limitation of magnetoelastic sensitivity of the torque sensors based on amorphous alloy strips [4].

To overcome these problems, the idea of new method of application of torque to ring-shaped sensing elements was developed [5]. The device utilizing this idea is presented in Fig. 1. Direction of the torque moment $T_{\mathrm{s}}$ is parallel to the axis of the ribbon ring-shaped core; as a result, shear stresses $\tau$ are both parallel and perpendicular to the magnetizing field $H$ (generated by magnetizing circuit).

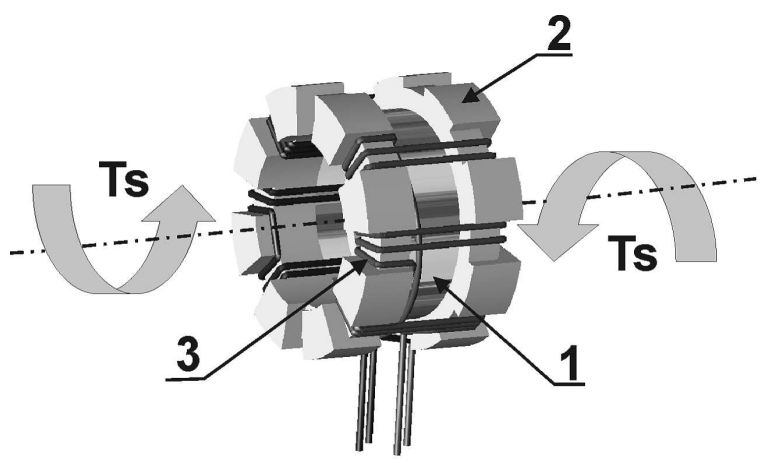

Fig. 1. Device for application of the torque to magnetoelastic ring-shaped core [5]: 1 - magnetoelastic sensing core, 2 - non-magnetic backings with grooves for winding, 3 - magnetizing and sensing windings.

In presented device, the ring-shaped sensing core (1) is mounted to the base planes of the special nonmagnetic backings (2), and the torque $T_{\mathrm{s}}$ is applied in the direction of axis of the ring. The nonmagnetic backings (2) have radial grooves, which enable core to be winded by magnetizing and sensing windings (3). As a result, the changes of sensing element's parameters under the influence of the torque $T_{\mathrm{s}}$ can be measured.

To confirm the uniform distribution of shear stresses in the sensing element subjected to the torque $T_{\mathrm{s}}$, the thermovision tests were performed. These tests indicated that the maximal shearing stress non-uniformity is smaller than $5 \%$.

The measurements of the influence of torque on the quasi-static hysteresis loops were carried out on the two ring-shaped cores, made of Hitperm-type $\mathrm{Fe}_{41.5} \mathrm{Co}_{41.5} \mathrm{Cu}_{1} \mathrm{Nb}_{3} \mathrm{~B}_{13}$ alloy. The outside diameter of cores was $32 \mathrm{~mm}$, inside diameter was $25 \mathrm{~mm}$, and height was $10 \mathrm{~mm}$. First core was in an amorphous as-quenched state, whereas second core was subjected to the thermal treatment at the temperature of $360^{\circ} \mathrm{C}$, for 1 hour. 


\section{Results}

The results of testing of the influence of torque $T_{\mathrm{S}}$ on hysteresis loop of Hitperm-type alloy, in as-quenched and nanocrystalline state, are presented in Fig. 2. It should be indicated that no significant influence of the torque $T_{\mathrm{s}}$ on the shape of hysteresis loop was observed, in the case of as-quenched sample. However, nanocrystalline samples exhibit stress sensitivity. Under the torque $T_{\mathrm{s}}$ up to about $4 \mathrm{~N} \mathrm{~m}$, flux density $B$ as well as coercive field $H_{\mathrm{c}}$ increased.

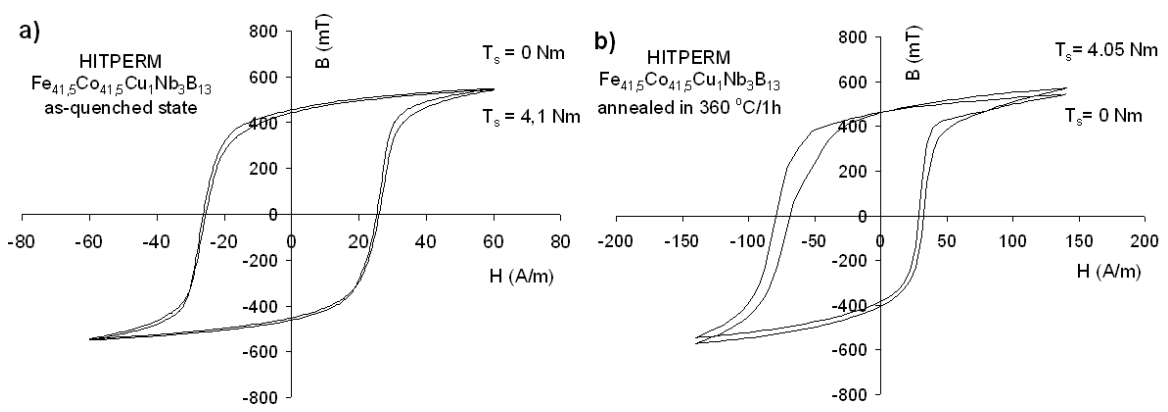

Fig. 2. The influence of the torque on hysteresis loop Hitperm-type $\mathrm{Fe}_{41.5} \mathrm{Co}_{41.5} \mathrm{Cu}_{1} \mathrm{Nb}_{3} \mathrm{~B}_{13}$ alloy in: (a) as-quenched state, (b) nanocrystalline state.

The changes of the flux density $B$ of the investigated cores made of Hitperm-type $\mathrm{Fe}_{41.5} \mathrm{Co}_{41.5} \mathrm{Cu}_{1} \mathrm{Nb}_{3} \mathrm{~B}_{13}$ alloy, in both as-quenched and nanocrystalline state, subjected to torque $T_{\mathrm{s}}$, are presented in Fig. 3. Dependences were determined as the function of torque $T_{\mathrm{s}}$, where flux density $B$ was measured for constant values of the magnetizing field $H_{\mathrm{m}}$.
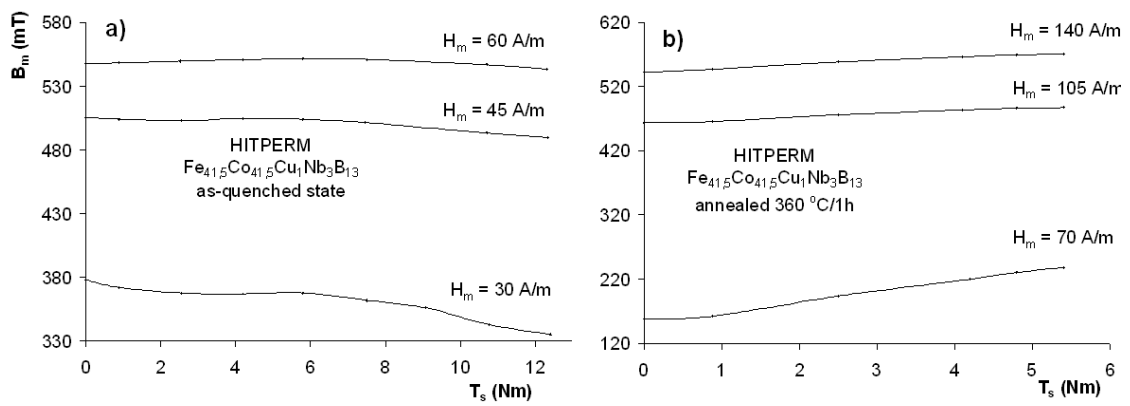

Fig. 3. The influence of the torque $T_{\mathrm{s}}$ on the flux density $B$ for (a) in as-quenched state and (b) after annealed state.

The presented results indicate that for higher values of magnetizing field $H_{\mathrm{m}}$, both as-quenched and nanocrystalline samples exhibit limited stress sensitivity. On the other hand, for lower values of magnetizing field $H_{\mathrm{m}}$, close to coercive field $H_{\mathrm{c}}$, for as-quenched sample, flux density $B$ decreases by about $12 \%$ under torque 
$T_{\mathrm{s}}$ up to $4 \mathrm{~N} \mathrm{~m}$. For the same torque $T_{\mathrm{s}}$ and magnetizing field near the coercive field $H_{\mathrm{c}}$, flux density $B$ in nanocrystalline sample increases about $36 \%$.

\section{Conclusion}

Due to the uniform distribution of shearing stress, the presented device for application of the torque to the ring-shaped cores made of amorphous or nanocrystalline alloy creates the new possibilities of the construction of the torque sensors, as well as fundamental research on magnetoelasticity. The presented results indicate that for higher values of magnetizing field $H_{\mathrm{m}}$, both as-quenched and nanocrystalline samples exhibit limited stress sensitivity. For lower values of magnetizing field $H_{\mathrm{m}}$, close to coercive field $H_{\mathrm{c}}$, under torque $T_{\mathrm{s}}$ up to $4 \mathrm{~N} \mathrm{~m}$ for as-quenched sample, flux density $B$ decreases by about $12 \%$, whereas in nanocrystalline sample increases by about $36 \%$. These results confirm that nanocrystallization process may change not only the value of stress sensitivity, but also the course of changes of flux density $B$ under the torque $T_{\mathrm{s}}$.

\section{Acknowledgments}

This work was partially supported by Polish Ministry of Science and Higher Education, within research grant realized in years 2005-2008.

Authors express their gratitude to Dr Roman Kolano of Institute of Non-ferrous Alloys for samples preparation.

\section{References}

[1] G. Herzer, Scr. Metallurg. 33, 1741 (1995).

[2] R. O'Handley, Modern Magnetic Materials - Principles and Applications, Wiley, New York 2000.

[3] I. Sasada, M. Inoue, A. Hiroike, K. Harada, IEEE Trans. Magn. MAG-19, 2148 (1983).

[4] E. Tremolet, Magnetostriction, CRC Press, London 1992.

[5] J. Salach, A. Bieńkowski, R. Szewczyk, Patent Pending, P-370124 (2004). 